\title{
Enhancing Handoff Performance By Introducing Ad hoc Mode into Cellular Networks
}

\author{
Rongsheng Huang, Student Member, IEEE, Chi Zhang, Student Member, IEEE, Hongxia Zhao, \\ and Yuguang Fang, Fellow, IEEE
}

\begin{abstract}
As all-IP feature becomes dominant in the next generation networks (NGNs), ad hoc mode is gaining more attention as an appealing addition to cellular networks. Consequently, multi-hop handoffs become inevitable, which bring challenging issues to network designers. Handoff dropping (HOD) rate and the bandwidth reservation for the required HOD rate are two important metrics to evaluate the handoff performance of a cellular system. By introducing ad hoc mode into cellular systems, we can either achieve lower HOD rate or reserve less bandwidth for the same required HOD rate. In this paper, we incorporate traffic information from neighboring BSs and propose an algorithm to find the minimum bandwidth reservation for each BS. Since its performance greatly depends on the access probability to the adjacent cells, we further propose to utilize the embedded ad hoc networks to gather the traffic load information of neighboring cells. With such information, handoff calls can effectively select the best paths to the proper BSs. It has been demonstrated that our scheme can significantly improve the system performance.
\end{abstract}

Index Terms-Cellular networks, Ad hoc networks, Mobility management, Handoff

\section{INTRODUCTION}

Multi-hop cellular networks (MCNs) have received growing attention lately due to the intention that the next generation cellular networks can provide an all-IP platform, where new types of wireless networks, e.g., WiFi and WiMAX, and traditional cellular networks can integrated seamlessly. 3GPP has been engaged in the study of this trend [1] and the specification of the interworking between WCDMA networks and WLANs was standardized years ago [2]. It is now wellknown that these new wireless networks, usually operated in ad hoc mode, can be constructed more flexibly with much lower cost and higher data rate, though the coverage and mobility cannot be well supported. Consequently, it is expected that MCNs can take advantage of both traditional (single-hop) cellular networks and the new types of wireless networks.

MCNs are commonly considered as cellular-based integration of cellular networks and ad hoc networks because ad hoc mode is an additive component to cellular systems, which does not impair the traditional cellular structure. This means that mobile users can access BSs through either direct (one-hop) connections or multi-hop connections.

R. Huang, C. Zhang and Y. Fang are with the Department of Electrical and Computer Engineering, University of Florida, Gainesville, FL 32611, USA. H. Zhao is with Huawei Technologies Co., Ltd. Tel: (352)392-8576; Fax: (352)392-0044, Email: \{rshuang@,Zhangchi@,fang@ece.\}ufl.edu.

This work was supported by the National Science Foundation under grants CNS-0721744.
Cavalcanti et al. provided a survey on these integrated networks [3]. Some of the features of the related works are compared. With the cellular-based multi-hop cellular networks under consideration, the first advantage over traditional cellular systems is the low cost coverage extension. Multi-hop wireless relay can easily provide faraway wireless terminals with connections without building extra infrastructures like BSs. Since the relay devices are usually much more portable than BSs, flexibility is the second expected advantage, leading to more efficient resource utilization. Wu et al. proposed iCAR, in [4], which requires special relay equipments at specified positions and hence lowers the call-blocking probability. Finally, with the ad hoc links bearing higher data rate and potentially operated on unlicensed band, the capacity of each cell can be expected to improve. UCAN is a unified cellular and ad-hoc network architecture based on 1xEV-DO (HDR) and 802.11b [5]. Here, by allowing wireless clients to relay the downlink traffic, the system can achieve better performance in terms of higher downlink data rate. ODMA can be found in 3GPP documents [6], which uses a similar scheme as [5].

In this paper, we attempt to investigate the benefits that the ad hoc mode can bring to the handoff performance. Handoff performance is crucial to cellular systems in keeping seamless connections. Handoff dropping (HOD) rate is the major metric of handoff performance. With the introduction of multi-hop connections, handoff processes can become more complicated. When an MN (Mobile Node) moves from one cell to another and chooses the new BS as its serving BS, the handoff process is no different from that in traditional cellular networks. Only when a multi-hop connection exists either before or after an MN alters its connection, do the handoffs face the new type of network design challenges. Cho et al. illustrated different types of handoffs in [7]. As a supplement to cellular systems, ad hoc links increase opportunities for MNs to connect to BSs. Therefore, HOD performance is expected to improve with ad hoc mode introduced in cellular systems. In traditional single-hop cellular networks, HOD rate is usually guaranteed by bandwidth reservation for handoff calls. Larger reservation does meet HOD rate requirement, however, at the price of sacrificing the call blocking rate because less resource is left for new calls. With the introduction of ad hoc mode, handoff calls may use adjacent cells' reservation as well as that in its current cell. When multiple handoff attempts are allowed, the actual HOD rate for each $\mathrm{MN}$ can be in fact reduced. To meet certain HOD rate requirement, BSs can reserve less bandwidth for handoff calls than before. In this paper, we present an algorithm to find the feasible minimum bandwidth 
reservation according to neighboring cells' information. By the use of the neighboring cells' traffic information, we provide a new way to design the resource management scheme to deal with handoff issues.

We observe that the performance of the above algorithm greatly depends on the access probability for handoff calls to the adjacent cells. Previous works have not provided effective ways to increase the access probability with existing relay devices. Special relay devices are suggested to be placed along the cell boundaries in [4] and [7]. Network planning is necessary for better handoff performance. Hence, one of the most important benefits of introducing the self-organizing ad hoc mode is neglected and the flexibility that ad hoc mode can offer has not been effectively utilized. In this paper, we propose a scheme to use the embedded, self-organizing small-scale ad hoc networks to assist the handoffs. We do not need relay nodes to be specifically fabricated, nor to be placed at certain locations. Rather, by exchanging traffic information inside the embedded ad hoc networks, relay nodes can cooperatively help handoff calls choose better handoff options and thus increase access probability and decrease the call dropping rate.

The rest of this paper is organized as follows. Section II presents the algorithm for finding the minimum reservation in multi-hop cellular systems and the scheme for increasing the access probability for handoff calls to adjacent BSs. Section III provides the performance evaluation. Conclusions are given in the final section.

\section{The Proposed SCHEMES}

\section{A. System Model}

There are two kinds of links in MCNs. One is the traditional cellular links which use the cellular spectrum. The other one is the ad hoc links which may use different spectrum and follow ad hoc protocols like IEEE802.11. The service area is usually divided into cells like traditional cellular systems where ad hoc links can cross the boundaries of adjacent cells. In the model of this paper, all MNs belong to the same system and each $\mathrm{MN}$ is always associated with one certain $\mathrm{BS}$, no matter whether it is connected via cellular links or via multi-hop ad hoc links. Each BS reserves a certain amount of bandwidth for the possible handoff traffic according to the predefined handoff/new call ratio.

We call the handoffs involving multi-hop connections as multi-hop handoffs. Fig. 1 illustrates the multi-hop handoffs in an MCN. In Fig. 1, MN A moves from cell 1 to cell 0. Unfortunately, the BS in cell 0 has no spare spectrum (The cell with a question mark has no spare spectrum for handoff calls). In the illustrated scenario, MN A has two choices. It can either access the BS 5 through MN B or access BS 7 through MN C and MN D. Either option can make the MN access to the system.

Since the direct connection to BSs has lower call interruption, each $\mathrm{MN}$ will always attempt the direct connection first. For new calls, MNs search for all possible connections until they succeed. For handoff calls, due to the time limit of the signaling process, MNs make several attempts to connect to different BSs until they either succeed or the time limit is reached. The connected MNs are responsible for relaying the connecting MNs' requests to the destination BSs. The destination BSs allocate cellular spectrum resource to the connecting MNs in care of the last-hop. The BSs keep the information of the relay relationship of its serving MNs in order to deliver packets correctly.

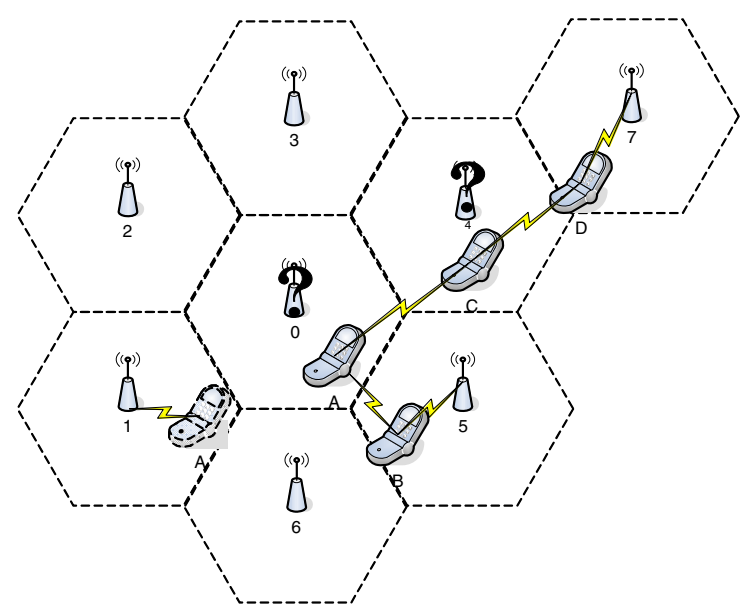

Fig. 1. Model of Multi-hop handoffs

\section{B. Finding The Minimum Reservation}

1) One-attempt HOD rate: Similar to traditional cellular systems, a given HOD rate is the input of a reservation scheme in MCNs. This input determines the expected HOD rate for each $\mathrm{MN}$ when it is physically within a certain cell. If there is only one cell's resource under consideration, according to Markov Chain model in previous papers [8], [9], the HOD probability can be obtained when the traffic arrival and departure rate are given. We denote the HOD rate when only one cell's resource is considered as $P_{0}$. The arrival rates of new calls and handoff calls are denoted as $\lambda_{N}$ and $\lambda_{H O}$, respectively. The departure rate of all calls is denoted as $\mu$. $R s v$ is the number of reserved channels. $C$ is the number of total channels. According to the model in [8], [9], we can derive the HOD rate as follows (more details can be found in the Appendix).

$$
\begin{aligned}
& P_{0}=\frac{\left(\lambda_{N}+\lambda_{H O}\right)^{C-R s v} \lambda_{H O}^{R s v}}{C ! \mu^{C}} \cdot\left(\sum_{k=0}^{C-R s v} \frac{\left(\lambda_{N}+\lambda_{H O}\right)^{k}}{k ! \mu^{k}}\right. \\
& \left.+\sum_{k=C-R s v+1}^{C} \frac{\left(\lambda_{N}+\lambda_{H O}\right)^{C-R s v} \lambda_{H O}^{k-C+R s v}}{k ! \mu^{k}}\right)^{-1}
\end{aligned}
$$

2) Algorithm of Finding Minimum Reservation: When multi-hop connections are available and multiple attempts are allowed, the HOD rate for the MNs can be further reduced. We denote the required HOD rate for each $\mathrm{MN}$ in certain cell as $P_{r}(H O D)$. Our goal is to find the minimum feasible reservation $R s v$ to meet the requirement of $P_{r}(H O D)$.

In the proposed algorithm to find the minimum reservations, we take into account the multiple attempts of multi-hop handoffs, which may lead to more successful access to adjacent 
cells. Therefore, periodic information exchange is required among adjacent BSs. To simplify the analysis, each BS only considers the multi-hop handoffs to its one-hop neighbors. BSs use the past measured information as the predictive value of next time interval. According to the previously measured values of arrival rate $\lambda$ and departure rate $\mu$, and the current reservation $R s v$, each $\mathrm{BS}$ calculates the one-attempt HOD rate and broadcast this value. Note that the measured arrival traffic $\lambda_{H O}$ consists of the original handoff traffic and the handoff traffic rejected in adjacent cells. The broadcasted value of oneattempt HOD rate from $\mathrm{BS} i, \tilde{P}_{i}$, is taken as the input of calculating the minimum reservation. Besides the HOD rate of neighboring cells, each BS also needs to know the probability that multi-hop handoffs can access the adjacent cell. This access probability, corresponding to a certain surrounding BS $i$, denoted as $q_{i}$, can be measured by dividing the number of MNs in current cell (BS 0) which have access to BS $i$ to the total number of MNs in current cell. Obviously, obtaining this information requires MNs to report extra information to $\mathrm{BS}$, the accessibility to other BSs.

With these broadcasted $\tilde{P}_{i}$ and measured $q_{i}$, we can derive the minimum reservation by incorporating formula (1), as in Eqn. (2). Here $N$ is used to denote the maximum number of attempts after the direct handoff attempt is failed and $M$ to denote the number of neighbors of the current cell.

$$
\begin{aligned}
& P_{r}(H O D)=P_{0}\left(\prod_{i=1}^{M}\left(1-q_{i}\right)+\sum_{i=1}^{M} \frac{\tilde{P}_{i}}{M} q_{i} \prod_{l \neq i}^{M}\left(1-q_{l}\right)\right. \\
& \quad+\sum_{i=1}^{M} \sum_{j \neq i}^{M} \frac{\tilde{P}_{i} \tilde{P}_{j}}{2 ! C_{M}^{2}} q_{i} q_{j} \prod_{l \neq i, j}^{M}\left(1-q_{l}\right)+\ldots+ \\
& \left.\sum_{i_{1}=1}^{M} . . \sum_{i_{N} \neq i_{1}, . . i_{N-1}}^{M} \frac{1}{N ! C_{M}^{N}} \prod_{k=1}^{N} \tilde{P}_{i_{k}} q_{i_{k}} \prod_{l \neq i_{1}, . . i_{N}}^{M}\left(1-q_{l}\right)\right)
\end{aligned}
$$

Note that in Eqn. (2), the required HOD rate is simply the summation of the probabilities that after a handoff fails in the current cell, it fails in a number of other cells. Each adjacent cell is assumed to be accessed randomly for analysis purpose.

With this equation, the minimum reservation can be found. However, the changed reservation will cause the change of local one-attempt HOD rate and then the one-attempt HOD rates in adjacent cells. New rounds of calculation will incorporate these changes and the new measured traffic load.

3) Impact of the Access Probability: In real multi-hop cellular networks, a rejected handoff call can attempt to connect to not only the adjacent BSs but also BSs further away, as long as the multi-hop paths exist. Eqn. (2) considers only the adjacent BSs in order to simplify the derivation of the minimum reservation.

Let us revisit Eqn. (2). When each $q_{i}$ has value 1, which means MNs in the current cell can access the resource of all the adjacent cells, this system can be seen as a larger cell consisting of all adjacent cells with aggregated resource from all adjacent cells. Obviously, this system has higher trunking efficiency which can lower call dropping rate and the requirement of reservation. When more than one hop neighboring cells are considered, even higher trunking efficiency and lower call dropping rate can be achieved.

However, the calculation outcome greatly depends on the access probabilities $q_{i}$ to neighboring cells. Lower $q_{i}$ cannot improve the reservation too much. The following proposed scheme aims at increasing the accessing probability to neighboring cells, $q_{i}$, and to further neighboring cells.

\section{Enhance the Multi-hop Handoff Possibility}

Multi-hop connections can reduce the HOD rate greatly as expressed in Eqn. (2). How to increase the neighboring cells' access probability and how each $\mathrm{MN}$ intelligently chooses better connection are two important ways to reduce the actual HOD rate. However, there is no previous work touching upon these issues.

In next generation networks, each $\mathrm{MN}$ can be a potential relay node thanks to the development of wireless techniques. Consequently, more potential paths of multi-hop connections exist among these nodes and higher probability of accessing neighboring cells can be achieved. Although choosing good connections can decrease the call dropping rate, choosing bad ones can lead to the opposite. Careless designs of handoff decision may increase the handoff frequency and increase the call dropping rate, leading to inefficiency of resource usage. Handoff decision becomes a more complicated task than purely relying on the signal strength. In this paper, we propose to utilize the embedded small-scaled ad hoc networks to assist the decision making for the handoff MNs.

As we all know, information can be effectively exchanged with the power of networking. Mobile Ad hoc Networks (MANETs) are well-researched areas where we have already had many results. To make the effect of information exchange less transient and to make this information more helpful to the handoffs, stationary or slow nodes are more preferred to form these handoff assisting networks. Obviously, when the scale of these assisting networks becomes large, the coverage can be wider and the handoff can receive more help. However, the maintenance of the assisting networks are becoming more difficult and the information exchange is less effective. Thus, we can employ stationary/semi-stationary, small-scale ad hoc networks to assist the multi-hop handoffs in cellular systems.

How to choose nodes and how to form these assisting networks is a very complicated problem which will be addressed separately. In this paper, to focus on the handoff issues, we simply assume that these networks can be formed by the instruction of operators. The small-scaled ad hoc networks dynamically update the information on available bandwidth, including the available path capacity and the expected value of available bandwidth in the cell. The latter can be derived according to the Markov chain model as [8], [9], given the arrival rate $\lambda$ and departure rate $\mu$ of one cell. In Eqn. (3), EstCellBW $W_{i}$ denotes the expected value of available bandwidth in the cell $i$. $a v g_{-} b w_{i}$ denotes the average bandwidth per user in cell $i$. $P(k)_{i}$ denotes the probability of $k$ users occupying cell $i$, the derivation of which can be found in 


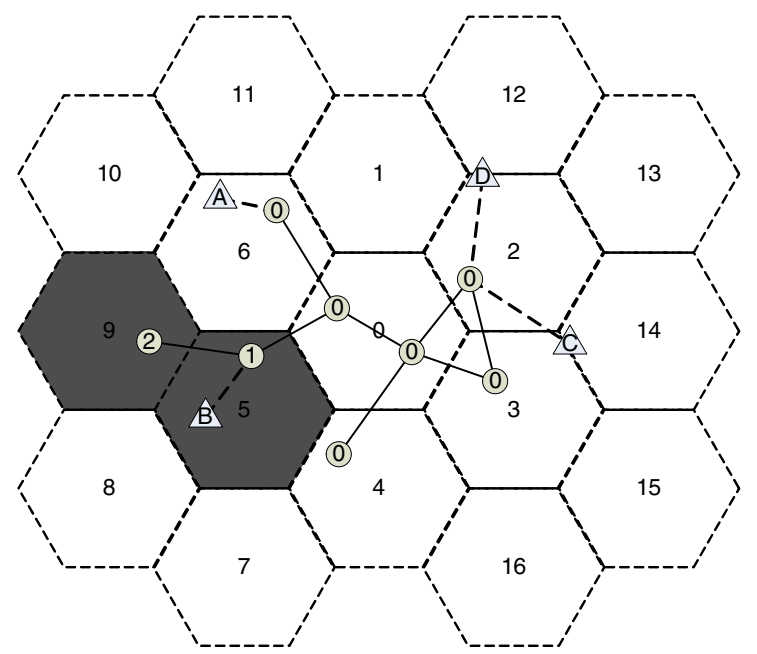

Fig. 2. Embedded Ad hoc Network in Cellular System

Appendix.

$$
\text { EstCellBW } W_{i}=\sum_{k=0}^{C} k \cdot P(k)_{i} \cdot a_{0} g_{-} b w_{i}
$$

For each attaching node, it compares all candidates' achievable bandwidth and expected call dropping rate and makes the decision. The ad hoc networks are required to further update the bandwidth information upon these decisions.

Besides the required bandwidth, the criterion on selecting a good strategy for multi-hop handoffs is to estimate the call dropping rate. We can have the call dropping rate expressed in such a formula as follows.

$$
P(\text { dropping })=1-(1-P(\text { con_fail })) \times(1-P(H O D))
$$

$P(H O D)$ is the probability of call dropping due to handoff failures. $P\left(\right.$ con_fail $\left._{-}\right)$is the probability of call dropping caused by connection failures. As mentioned above in Eqn. (4), it includes not only the handoff dropping due to handoff failure, but also the call dropping a multi-hop handoff might bring. $P(H O D)$ is determined not only by the current handoff failure rate, but also the possible incurred extra handoffs. For multihop handoffs, handoff failures can be caused by the failure of changing BSs, changing portal nodes, or changing docking nodes. Here the portal nodes mean the relay nodes directly connecting to the BSs and the docking nodes mean the relay nodes directly connecting to the handoff nodes. By comparing all the candidate paths of connections, each $\mathrm{MN}$ chooses the one which not only meets the bandwidth requirement, but also has the lowest $P$ (dropping).

\section{Performance Evaluation}

In this section, we study the benefits that multi-hop connections can bring to the cellular systems in terms of handoff performance.

Firstly, we look into the HOD rate improvement when reservation of each BS remains unchanged. We set a scenario of seven adjacent cells with one cell in the center and six cells surrounding it. For the single cell HOD rate, we calculate it
TABLE I

Simulation Setting For EACh Cell

\begin{tabular}{|l|l|}
\hline Number of Channels & 30 \\
\hline Number of Reserved Channels & 5 \\
\hline Average Number of Initial Calls & 40 \\
\hline Average Number of Handoff Calls & $120 \sim 270$ \\
\hline Access Probability & $0 \sim 1$ \\
\hline Average Call Intensity per User & $0.1 \mathrm{erl}$ \\
\hline
\end{tabular}

according to Eqn. (1). The detailed derivation comes from the model in [8], [9]. The basic setting of resource parameters is listed in Table I.

Each BS calculates the overall HOD rate according to Eqn. (2) with the knowledge of its adjacent BSs' singlecell HOD rate. The access probability to the neighboring cells are set as different identical constants. This calculation gives out the statistical HOD rate that all the MNs in the BS experience. The choice of multi-hop handoffs are assumed randomly made. With the proposed scheme, MNs are expected to achieve higher performance since more knowledge about handoff choices are handy and the intelligent handoff decision algorithm can greatly reduce the repeated attempting traffic.
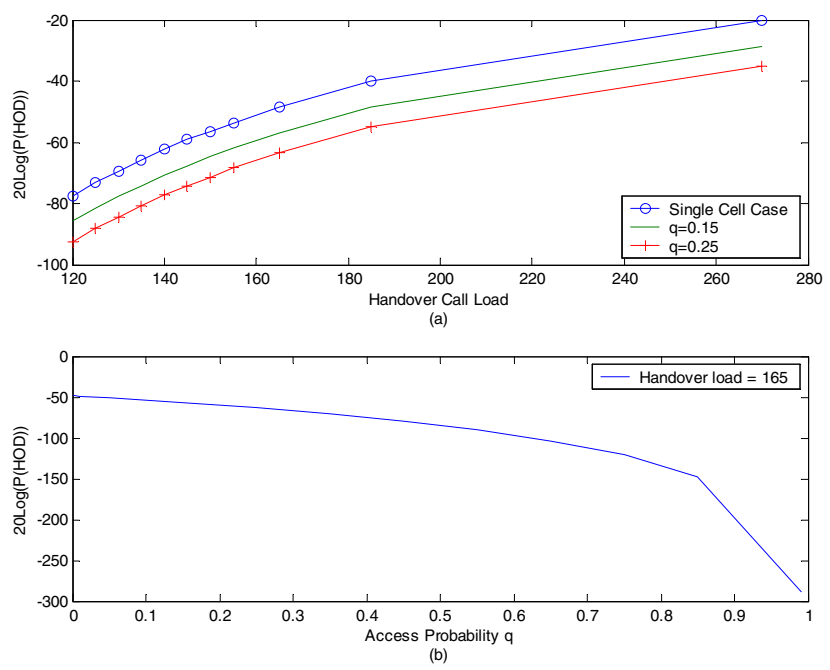

Fig. 3. HOD rate Improvement

In Fig. 3, the overall HOD rate improvement of the central cell has been shown. Part (a) shows the relationship between traffic load and HOD rate. From Part (a), we can see that when traffic load increases, the HOD rate increases accordingly. With the help of adjacent cells, HOD rate can be improved greatly. From Part (a), we can see that even with a small access probability, such as $q=0.25$, the HOD rate can be improved with 20db. We can also see from Part (a), when traffic load increases to a certain level, the HOD rate will deteriorate. The reason is that the current reservation cannot support the traffic load and the rejected traffic also forms a heavy burden to the adjacent cells. Part (b) shows the relationship between HOD rate and different access probabilities under a certain traffic load. We observe that when access probability increases, the HOD rate increases dramatically. 

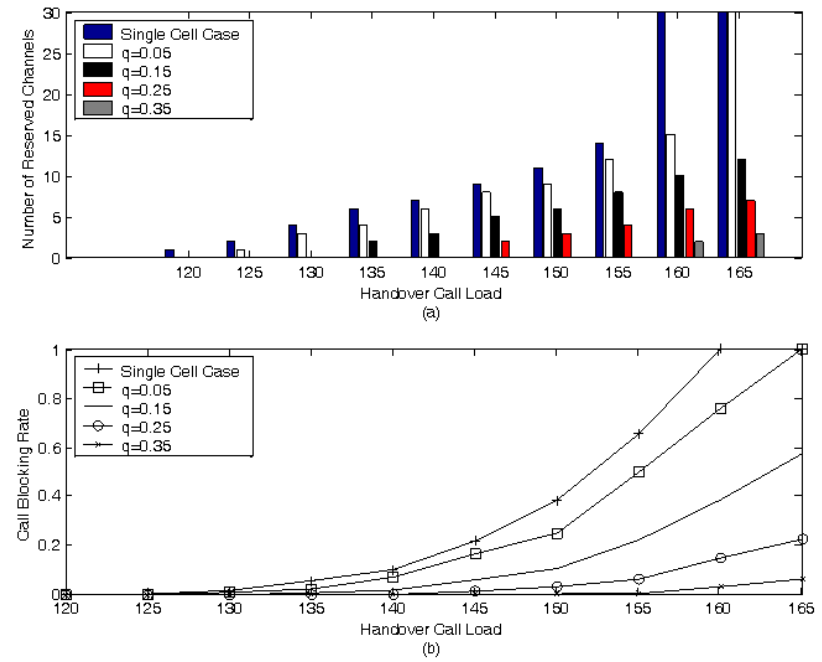

Fig. 4. Channel Reservation Improvement

Secondly, we evaluate how the reservation requirement is relieved with the help of adjacent cells. Lower reservation can also greatly reduce the call blocking rate of new calls. In this part of simulation, we set the required HOD rate as $0.5 \times 10^{-3}$. Under this constraint, each BS finds the minimum channel reservation. In Part (a) of Fig. 4, the numbers of reserved channels are shown with different traffic loads. We can see that without the help of adjacent cells ("Single Cell Case" in the figure), more channels need to be reserved to achieve the required HOD rate. When traffic load becomes larger, reservation does not work for the required HOD rate. In Part (a) of Fig. 4, simulations with 30 reserved channels stand for this case. With the access probability increasing, the reservation greatly decreased. In the case of $q=0.35$, under most of the traffic load, there is no need for reservation to meet the HOD rate requirement. Part (b) of Fig. 4 shows the corresponding call blocking rate when the minimum reservation is applied. We can easily see the great improvement especially when traffic load becomes heavier.

\section{CONCLUSION}

Ad hoc links can offer great improvements to cellular systems in terms of handoff performance since they provide additional multi-hop connections to BSs. This paper delves into how the reservation can be reduced with the existence of alternative connections to adjacent cells. Upon achieving the required HOD rate, with the existence of multi-hop connections, each BS can reduce the channel reservation for handoff calls, thus reduce the blocking rate of new calls. The proposed scheme utilizing the embedded ad hoc networks to assist the multi-hop handoffs also shows novel thoughts to reduce HOD rate by increasing access probability and intelligently choosing connections with lower potential HOD rate.

\section{APPENDIX}

According to [8], [9], we can use the following Markov chain model for the reservation system, where $S_{i}$ means the state of $i$ channels being occupied. $\lambda_{N}$ and $\lambda_{H O}$ stand for the arrival rate of new calls and handoff calls, respectively. $C$ is the total number of channels and $R$ is the number of reserved channels. $\mu$ is the departure rate for both types of calls. In this model, handoff calls and new calls start to use the shared channels. When the shared channels are used up, only handoff calls can use the reserved channels.

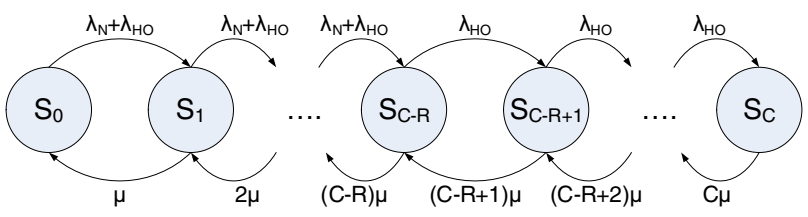

Fig. 5. Markov Chain Model for Handoff Reservation System

We then can write the state equations as follows.

$P(i)= \begin{cases}\frac{\lambda_{N}+\lambda_{H O}}{i \mu} \cdot P(i-1), & \text { for } i=1,2, \ldots, C-R \\ \frac{\lambda_{H O}}{i \mu} \cdot P(i-1), & \text { for } i=C-R+1, \ldots, C\end{cases}$

With the normalization condition, we can derive the probability of State 0 .

$$
\begin{aligned}
P(0) & =\quad\left(\sum_{k=0}^{C-R} \frac{\left(\lambda_{N}+\lambda_{H O}\right)^{k}}{k ! \mu^{k}}\right. \\
& \left.+\quad \sum_{k=C-R+1}^{C} \frac{\left(\lambda_{N}+\lambda_{H O}\right)^{C-R} \lambda_{H O}^{k-C+R}}{k ! \mu^{k}}\right)^{-1}
\end{aligned}
$$

The HOD happen when all the channels are occupied. Therefore, the HOD rate is the probability of State $C$, i.e., $P(C)$. The call blocking probability equals to the summation of probabilities of from State $C-R$ to State $C: P_{B}=$ $\sum_{k=C-R}^{C} P(k)$.

\section{REFERENCES}

[1] 3GPP Group. All-IP network (AIPN) feasibility study. 3GPP TS 22.978 V7.1.0.

[2] 3GPP Group. 3GPP system to wireless local area network (WLAN) interworking. 3GPP TS 23.234 V7.7.0.

[3] D. Cavalcanti, D. Agrawal, C. Cordeiro, B. Xie, and A. Kumar. Issues in integrating cellular networks WLANs, and MANETs: a futuristic heterogeneous wireless network. Wireless Communications, IEEE, 16(3):30-41, Jun 2005.

[4] H. Wu, C. Qiao, S. De, and O. Tonguz. Integrated cellular and ad hoc relaying systems: iCAR. IEEE Journal on Selected Areas in Communications (JSAC), 19(10):2105-2115, October 2001.

[5] H. Luo, R. Ramjee, P. Sinha, L. Li, and S. Lu. UCAN: A unified cellular and ad-hoc network architecture. In Mobicom'03, San Diego, CA, Sep 2003.

[6] 3GPP Group. Opportunity driven multiple access. 3G TR 25.924 version 1.0.0.

[7] S. Cho, E. Jang, and J. Cioffi. Handover in multihop cellular networks. IEEE Communication Magazine, 47(7):64-73, July 2009.

[8] D. Hong and S. Rappaport. Traffic model and performance analysis for cellular mobile radio telephone systems with prioritized and nonprioritized handoff procedures. IEEE Transactions on Vehicular Technology (TVT), 35(3):77-92, August 1986.

[9] Y. Fang and Y. Zhang. Call admission control schemes and performance analysis in wireless mobile networks. IEEE Transactions on Vehicular Technology (TVT), 51(2):371-382, March 2002. 